\title{
The Fragment-based Consistency Score in Model Quality Assessment for De Novo Prediction of Protein Structures
}

\author{
Hikmet Cetin ${ }^{1}$, Takeshi N Sasaki ${ }^{2}$, Masaki Sasai ${ }^{1,3,4^{*}}$ \\ ${ }^{I}$ Department of Computational Science and Engineering, Nagoya University, Nagoya 464-8603, Japan \\ ${ }^{2}$ Faculty of Human Informatics, Aichi Shukutoku University, Aichi 480-1197, Japan \\ ${ }^{3}$ School of Computational Sciences, Korea Institute for Advanced Study, Seoul 130-722, Korea \\ ${ }^{4}$ Okazaki Institute for Integrative Bioscience, Okazaki 444-8787, Japan \\ *E-mail:sasai@cse.nagoya-u.ac.jp
}

(Received August 31, 2011; accepted November 8, 2011; published online December 5, 2011)

\begin{abstract}
Prediction of three-dimensional structures of proteins from amino-acid sequences is an important problem of bioinformatics. We still do not have, however, a reliable computational method for de novo prediction, i.e., prediction of structures that do not have homologues of known structures. One way to improve techniques of de novo prediction is to assess quality of the predicted model structures to select candidate models from them before knowing the experimentally determined structure. In this paper, we develop a new method of model quality assessment for de novo prediction, the fragment-based consistency score (FCS) method. In the FCS method, fragments from library proteins are collected for each fragment of the model, and structural similarities between the model fragment and the library fragments are measured for assessing qualities of the model. Three structural indices are employed; secondary structure, local density and local contact order. The optimal performance was obtained when relatively correlated fragments are collected, and structural similarity is measured in fuzzy comparison and averaged by finite width matching. The FCS method can select partially correct models for hard targets of de novo prediction examined in CASP7 and CASP8. We also evaluated the abilities of the FCS method to select structural models of loops or coil regions in targets, and to distinguish proteins which are similar in sequence but have greatly different conformations from each other. The proposed FCS method helps to improve the de novo prediction scheme and is also useful to solve difficult structures in the template-based modeling.
\end{abstract}

Key Words: Structure Prediction, Quality Assessment, Loop Structure, Template Free Modeling

Area of Interest: Bioinformatics and Biocomputing 


\section{Introduction}

The problem to predict protein structures from amino acid sequences is a major challenge in bioinformatics. When the sequence of the target protein resembles sequences of proteins whose structures have been already resolved, we can use those known structures as templates for modeling the unknown target structure. Considerable advance has been made in techniques of template-based modeling (TBM) [1][2], but a systematic and consistent method has not yet been developed to predict target proteins which do not have a suitable template. The latter problem is called de novo prediction, or template-free modeling (FM), which is the harder problem than TBM but is important for applications in wider problems of structural biology and for understanding principles of protein architecture [3][4][5][6][7]. In order to improve the de novo prediction technique, a helpful approach is to use methods of model quality assessment (MQA or QA).

QA is the problem to estimate the similarity of the predicted model structures to the correct target structure before knowing the correct answer structure of the target. When many proposed model structures are available, a suitable QA should help to pick up the candidates from them, which could improve the performance of the prediction. Bartlett and Taylor [8], for example, have developed a QA method based on the pairwise correlation of residues between evolutionarily related sequences, and demonstrated that such QA helps to discriminate the incorrect structure models from those generated by a de novo prediction method. Importance of QA has become widely acknowledged, and since the seventh Critical Assessment of techniques for protein Structure Prediction (CASP7) [9], many QA methods have been compared in the QA session in CASP [10]. Through the analyses in the QA category in CASP, it has been shown [10][11][12] that the methods based on the common structural features, or the consensus among many predicted models [13][14][15][16][17][18][19][20][21][22], have given considerably better results than the methods which are not based on consensus. This is probably because errors in models generated by different prediction methods can be treated as noises which are filtered out through comparison of many models. The consensus-based methods, however, have their own limitations: When the majority of prediction methods failed but a few exceptional methods made good predictions, the consensus-based QA fails to select good models from the ensemble of proposed models. This problem should arise particularly when many prediction methods rely on the sequence alignment among the target and templates but the correct target structure does not resemble any of templates, which is the situation often encountered in FM problems. Furthermore, when we can use only a single or a small number of prediction methods, many models generated through the same method should have the same biased errors, so that the consensus-based methods may not work as well as in their use during CASP in which models of many different prediction servers are available. From these reasons, it is worth developing QA methods which do not rely on the consensus analyses. The combined use [16] of thus improved non-consensus-based method and the other consensus-based methods is expected to provide the further QA method which should improve the de novo prediction schemes. Though the non-consensus-based methods are often called the single-model methods in literatures [10][18][20][21][23], we here avoid this naming because we are not examining the ability of the method to assess quality of each individual model structure but we mainly focus on how the non-consensus-based method works to select good models from many structures.

Here, in this paper, we propose and test a new non-consensus-based QA method, the fragment-based consistency score (FCS) method, to improve the de novo prediction scheme. Many types of non-consensus-based QA methods have been developed by using the knowledge-based score functions derived from the sequence-structure correspondence [24][25][26], from the pairwise probability of residue distributions or contacts [27][28][29][30], or through the sequence 
alignment to utilize the homology among proteins [18][31][32]. In this paper, to mainly focus on the de novo prediction problem, we develop a knowledge-based score function without relying on the sequence homology relations.

Even in the FM problems in which the homology relation is not established between the target and other structurally resolved proteins, the local sequence-structure (1D-3D) relationships among multiple proteins should help to infer structures. This point has been most clearly demonstrated by the success of the fragment assembly method [7][33][34][35][36] and the method to assemble structural parts of variable length [37][38][39] in de novo prediction of FM targets. Here, the important feature in these methods is the use of collection of fragments with finite length to infer the local 1D-3D relationships instead of using single residues. In the same way as in these de novo prediction techniques, the QA method should also be improved by comparing finite-length fragments at around each residue. The fragment comparison has been used efficiently to derive the score function for QA [22], and in the present paper not only the structure of fragments but also the local structural environment around fragments are used to derive the 1D-3D relation. We should note that we only use the $\mathrm{C}_{\alpha}$ coordinates and the side-chain information is not used to calculate the FCS function in this paper.

The analysis is a straightforward extension of the 1D-3D method of VERIFY3D [24] and the related methods [25][40]. At each residue of the model, the local structural environment is classified in terms of structural indices, and the protein structure is represented by a string of structural indices, which we call the structure string. Around each residue, 9-residue-length fragments are selected from the library of non-redundant protein structures by consulting the sequence-profile table generated by the Position Specific Iterative (PSI)-BLAST [41]. Then, by counting the number of times of appearance of structural strings in the selected fragments, the fragment-based consistency score of the model structure is evaluated. Details of this FCS method are explained in Methods section.

There are various different ways of evaluating the fragment-based consistency score in the actual implementation of calculation. We compare these different calculation methods by using 18 domains of FM target proteins in CASP7 as references, and we show that the suitable averaging in evaluation of local scores is essential to obtain the best performing FCS. As a benchmark test, the method tuned in this way is applied to 12 FM targets in CASP8. It is also shown that the FCS method is useful for selecting models of long loops or the coil regions in the TBM targets in CASP8, and that the FCS method can distinguish structures of T0498 (PDB code: $2 \mathrm{kdl}$ ) and T0499 (PDB code: $2 \mathrm{kdm}$ ) in CASP8, which have the small difference in sequence but have the large difference in conformation [42], to which the consensus-based QA failed to give correct answers.

\section{Methods}

The FCS method is based on the ensemble of fragments selected from the structure library according to their similarity in sequence profiles to the sequence windows of the target protein. Then, to assess the quality of the model structure, the fragment-based consistency score is evaluated by comparing the structural features of fragments of library proteins and the structural features of the model. Within this overall scheme, there are various ways of calculating the fragment-based consistency score in actual evaluation. We explain those variations in this section and the difference in their performance is discussed in section 3.1. 


\subsection{Sequence profile}

Sequence profiles for the target protein and for proteins of the structure library are derived by comparing their sequences with those in the non-redundant (NR) sequence database [43] by 3 times iteration of PSI-BLAST [41] with the E-value cutoff of 0.001.

\subsection{Fragments}

Each of 9-residue windows in the sequence of the target protein is compared with all available 9-residue windows of sequences in the library of non-redundant protein structures which were selected from the list of Protein Data Bank (PDB) by using PISCES server [44][45] with its default parameters. For the fair comparison of our method with other QA methods reported in previous CASP opportunities, we selected 3624 library structures from the PDB data released before CASP7 to assess quality of model structures proposed in CASP7, and 5164 structures from the PDB data released before CASP8 to assess quality of model structures in CASP8.

The 9-residue window centered around the $i$ th residue of the target protein is denoted by $W(i)$ with $i=4, \ldots, N-4$, where $N$ is the total number of residues in the target. For every $W(i)$, 9-residue windows are selected from the structure library according to the correlation between the sequence profile of the window in the library proteins and the sequence profile of $W(i)$. The most correlated $N^{\text {fr }}$ windows are selected as fragments to be referenced for $W(i)$ and are denoted by $F_{k}(i)$, with $k=$ $1, \ldots, N^{\mathrm{fr}}$. We compare the following two ways to determine $N^{\mathrm{fr}}$.

(A) Collecting Correlated Fragments $(C F)$ : Fragments having the correlation coefficient larger than a threshold are collected. The threshold was calibrated to be 0.65 so as to maximize the performance in assessing quality of models of 18 FM targets in CASP7. The number of selected fragments is limited to be less than 380 . When the number of fragments having the correlation coefficient larger than 0.65 is smaller than 80, then 80 fragments of largest correlation are selected. In this way, $N^{\mathrm{fr}}$ depends on $i$ as $N^{\mathrm{fr}}(i)$ with $80 \leq N^{\mathrm{fr}}(i) \leq 380$.

(B) Collecting Relatively Correlated Fragments $(R C F)$ : Fragments whose correlation coefficients $C_{k}(i)$ is in the range $\eta C^{\mathrm{Top}}(i) \leq C_{k}(i) \leq C^{\mathrm{Top}}(i)$ are collected from the library, where $C^{\mathrm{Top}}(i)$ is the highest correlation coefficient between the sequence profile of $W(i)$ and the sequence profile of all available fragments in the library. The parameter $\eta$ was calibrated to be 0.75 so as to maximize the performance in assessing quality of models of $18 \mathrm{FM}$ targets in CASP7. The number of fragments is set to be $80 \leq N^{\mathrm{fr}}(i) \leq 380$ as in the $C F$ method explained above.

\subsection{Structure string}

The protein chain is expressed by the connected beads of $\alpha$ carbons, whose coordinates are denoted by $\left\{\mathbf{r}_{i}\right\}$. To calculate the fragment-based consistency score, the local structural environment is characterized by three indices. (1) Index of secondary structure, $S_{i}$; We define the dihedral angle $\theta_{i}$ between the plane spanned by $\mathbf{r}_{i-1}, \mathbf{r}_{i}$, and $\mathbf{r}_{i+1}$, and the plane spanned by $\mathbf{r}_{i}, \mathbf{r}_{i+1}$, and $\mathbf{r}_{i+2}$. The local structure around the $i$ th site is classified into $S_{i}=\alpha$ when $\alpha_{0}<\cos \theta_{i}, S_{i}=\beta$ when $\cos \theta_{i}<\beta_{0}$, and $S_{i}=$ $\mathrm{C}$ when $\beta_{0} \leq \cos \theta_{i} \leq \alpha_{0}$. The parameters $\alpha_{0}$ and $\beta_{0}$ were calibrated to reproduce the secondary structure assignment of the library proteins in a most reasonable way as $\alpha_{0}=0.35$ and $\beta_{0}=-0.8$. (2) Index of local density, $D_{i} ; N_{10}$ is defined by the number of $\alpha$-carbons in the sphere of radius $10 \AA$ around the position $\mathbf{r}_{i}$. We write $D_{i}=1$ when $N_{10} \leq 6, D_{i}=2$ when $7 \leq N_{10} \leq 12, D_{i}=3$ when $13 \leq$ 
$N_{10} \leq 18$, and $D_{i}=4$ when $19 \leq N_{10}$. (3) Index of local contact order, $L_{i}$; Local contact order (LCO) is defined by the average of distance along the sequence, $|i-j|$, between the centered $i$ th residue in the window and the other $j$ th residue located in the sphere of radius $10 \AA$ around the position $\mathbf{r}_{i}$. We write $L_{i}=1$ when LCO $\leq L_{0}$, and $L_{i}=2$ when LCO $>L_{0}$. We set $L_{0}=28.861$ to make the total number of residues with $L_{i}=1$ approximately equal to the total number of residues with $L_{i}=2$ for proteins in the structure library. In this way, the local structure around the $i$ th residue is represented by $x_{i}=\left(S_{i}, D_{i}, L_{i}\right)$. Using thus defined set of three indices, the protein structure is represented by a sequence of $\left\{x_{i}\right\}$ which we call the structure string.

\subsection{Fragment-based consistency score}

We introduce an index $p_{k}(i)\left\{x_{j}: x_{n}\right\}$ to compare structure strings, where $x_{j}=\left(S_{j}, D_{j}, L_{j}\right)$ is the local structure at the $j$ th residue of the model with $j \in W(i)$, and $x_{n}=\left(S_{n}, D_{n}, L_{n}\right)$ is the structure at the residue $n$ in $F_{k}(i)$. We adopt the following two ways of comparing structure strings.

(A) All or None Comparison (ANC): matching of all three indices is required, so that we set $p_{k}(i)\left\{x_{j}: x_{n}\right\}=1$ when $x_{j}=x_{n}$ and $p_{k}(i)\left\{x_{j}: x_{n}\right\}=0$ when $x_{j} \neq x_{n}$.

(B) Fuzzy comparison $(F C)$ : Different degree of structure matching is taken into account. We set $p_{k}(i)\left\{x_{j}: x_{n}\right\}=1$ when $x_{j}=x_{n}, p_{k}(i)\left\{x_{j}: x_{n}\right\}=0.5$ when two of three indices of $x_{j}$ are identical to those of $x_{n}, p_{k}(i)\left\{x_{j}: x_{n}\right\}=0$ when one of three indices of $x_{j}$ is identical to that of $x_{n}$, and $p_{k}(i)\left\{x_{j}\right.$ : $\left.x_{n}\right\}=-0.5$ when all three indices of $x_{j}$ are different from those of $x_{n}$. For example, $p_{k}(i)\left\{x_{j}: x_{n}\right\}$ $=0.5$ when $S_{j}=S_{n}, D_{j}=D_{n}$, and $L_{j} \neq L_{n}$, and $p_{k}(i)\left\{x_{j}: x_{n}\right\}=0$ when $S_{j} \neq S_{n}, D_{j}=D_{n}$, and $L_{j} \neq L_{n}$.

We write the center residue of the fragment $F_{k}(i)$ as $k_{0}$, and write the five consecutive residues around $k_{0}$ as $k_{0}-2, k_{0}-1, k_{0}, k_{0}+1$, and $k_{0}+2$. From $p_{k}(i)\left\{x_{j}: x_{n}\right\}$, we derive the local matching index $q_{k}(i)$ in the following two different ways..

(A) Center-to-Center Matching (CCM): Only the center of the sequence window of the model protein and the center of the fragment are compared, so that $q_{k}(i)=p_{k}(i)\left\{x_{i}: x_{k 0}\right\}$.

(B) Finite Width Matching ( $F W M)$ : The structural environment of residues in $W(i)$ is compared with fragments by setting $q_{k}(i)=p_{k}(i)\left\{x_{i}: x_{k 0}\right\}+0.5 p_{k}(i)\left\{x_{i+1}: x_{k 0+1}\right\}+0.5 p_{k}(i)\left\{x_{i-1}: x_{k 0-1}\right\}+$ $0.25 p_{k}(i)\left\{x_{i+2}: x_{k 0+2}\right\}+0.25 p_{k}(i)\left\{x_{i-2}: x_{k 0-2}\right\}$.

From $q_{k}(i)$, the local fragment-based consistency score for $W(i), L F C S_{i}$, is derived. We define the normalized weight factor of the $k$ th fragment as $f_{k}(i)=\left(C_{k}(i)-C^{\mathrm{Bottom}}(i)\right) /\left(C^{\mathrm{Top}}(i)-C^{\mathrm{Bottom}}(i)\right)$, where the correlation coefficient $C^{\text {Bottom }}(i)$ is the lowest correlation between the sequence profile of $W(i)$ and sequence profiles of $N^{\mathrm{fr}}(i)$ fragments. Then, $L F C S_{i}$ is calculated as

$$
\operatorname{LFCS}_{i}=\sum_{k=1}^{N_{\mathrm{ff}}^{\mathrm{f}}(i)} q_{k}(i) f_{k}(i) .
$$

The fragment-based consistency score of the model structure is obtained as

$$
F C S=\sum_{i=4}^{N-4} L F C S_{i}
$$


As described above, various different methods are possible to calculate the fragment-based consistency score in the actual implementation; $C F$ or $R C F, A N C$ or $F C$, and $C C M$ or $F W M$. In Results section, performance of these $2^{3}=8$ methods is compared.

\section{Results}

\subsection{Selecting the calculation scheme of FCS}

Variants of the FCS calculation method are compared with each other by using 18 FM domains as references. These 18 target domains are explained in Table 4 in Appendix. For each target, the quality of all models predicted by the server teams, i.e., teams attended in CASP7 to predict structures by using methods of automated computation, are assessed by these methods of FCS. The number of available server models is 640-940 depending on the target [46]. We write the fragment-based consistency score of the $a$ th model structure of the $n$th target protein calculated with the method designated by $\mu$ as $F C S(a, n, \mu)$ with $\mu=C F-A N C-C C M, R C F-A N C-F W M$, and so on, and $n=1-18$.

As a measure of structural similarity between the model structure and the correct answer structure, we adopt the Global Distance Test Total Score (GDTTS) [47]. Each prediction team attending the CASP experiment can submit 5 model structures to the CASP organizers [9]. Using this criterion also in this paper, we select 5 model structures which give the highest FCS of all server models for given $n$ and $\mu$. Then, the structure which has the largest GDTTS of 5 models is denoted by $a^{\text {best }}(n, \mu)$.

We write GDTTS of the $a$ th model structure of the $n$th target domain as $\operatorname{GDTTS}(a, n)$. Plotted in Figure 1 is the relative GDTTS, $\triangle G D T T S$, for different $\mu$,

$$
\Delta \operatorname{GDTTS}(n, \mu)=\frac{\operatorname{GDTTS}\left(a^{\text {best }}(n, \mu), n\right)-\operatorname{GDTTS}(\max )}{\operatorname{GDTTS}(\max )},
$$

where $\operatorname{GDTTS}(\max )$ is the maximum value of $\operatorname{GDTTS}\left(a^{\text {best }}(n, \mu), n\right)$ for $\mu$ examined in each panel of Figure 1. In Figure 1, curves of $\Delta \operatorname{GDTTS}(n, \mu)$ are plotted as functions of $n$ in the descendent order of $\Delta \operatorname{GDTTS}(n, \mu)$.

In Figure 1, the method showing a curve of the larger $\triangle G D T T S$ is the better method. From Figures $1 \mathrm{a}$ and $1 \mathrm{c}$, we can see that $F C$ consistently gives the better results than $A N C$. From Figures $1 \mathrm{a}$ and $1 \mathrm{~b}$, we see that $F W M$ gives better results than $C C M$ in general, and from Figures $1 \mathrm{~b}$ and $1 \mathrm{c}$, we find $R C F$ gives better results than $C F$. From the superiority of Fuzzy Comparison and Finite Width Matching, we find that the suitable averaging in calculating $L F C S_{i}$ is essentially important for obtaining the good performance. The best method is $\mu=R C F-F C-F W M$, and hence in the following, we use this method in applications.

\subsection{Local fragment-based consistency score}

Since FCS is a sum of LFCS as explained in Equation 2, it should be meaningful to examine the performance of LFCS before comparing FCS with other QA methods. We use $R C F-F C-F W M$ method chosen in the previous subsection. We selected four example targets out of 12 CASP8 FM targets, for which the best server models of CASP8 showed the relatively high GDTTS. In Table 1, five models having the highest FCS are examined for these four example targets. For each selected 

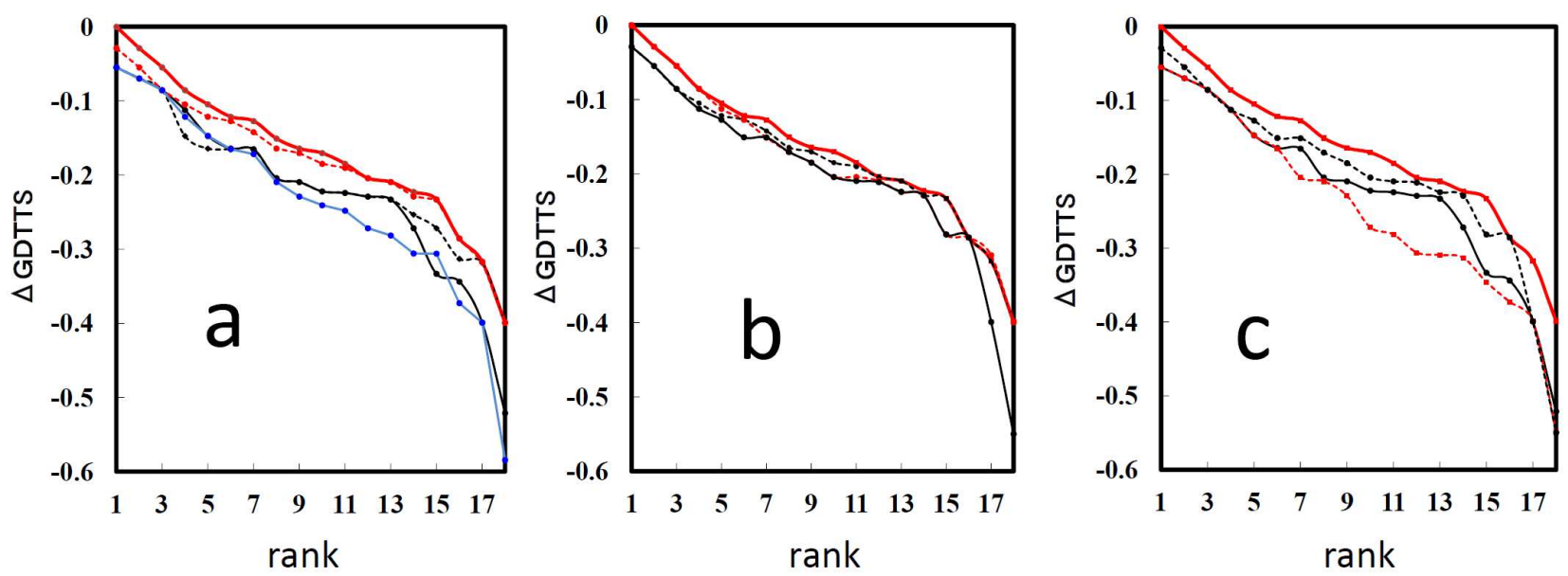

Figure 1. Comparison of various methods to calculate FCS

8 ways to calculate FCS are compared in terms of $\triangle G D T T S$ of 18 domains of FM targets in CASP7. In each panel, $\triangle G D T T S$ is plotted in the descendent order for 18 targets. FCS is calculated with methods of (a) $R C F-F C-F W M$ (red real line), RCF-ANC-FWM (black real line), $R C F-F C$-CCM (red broken line), $R C F-A N C-C C M$ (black broken line), and $C F-A N C-C C M$ (blue real line), (b) $R C F-F C-F W M$ (red real line), $C F-F C-F W M$ (black real line), $R C F-F C-C C M$ (red broken line), and $C F-F C$ - $C C M$ (black broken line), and (c) $R C F-F C-F W M$ (red real line), $C F-F C-F W M$ (black broken line), $R C F-A N C-F W M$ (black real line), and $C F-A N C-F W M$ (red broken line).

model, the Pearson correlation coefficient $P_{\text {model }}$ is defined between $R M S D_{i}$ and $L F C S_{i}$ for $i=4, \ldots$, $N-4$, where $R M S D_{i}$ is the root-mean-square-deviation (RMSD) of the $i$ th fragment structure from the correct answer structure. We can see that $P_{\text {model }}$ is high in models having the relatively large GDTTS. In this way, LFCS has the ability to discriminate local structures when the model contains good fragments. This ability for local structure assessment will be further tested in Subsection 3.4 by examining the performance of FCS to assess quality of the coil or loop regions of model structures.

\subsection{Comparisons with other QA methods}

By using the RCF-FC-FWM method, the FCS method is applied to the server models in CASP7 and CASP8 to compare the performance of the FCS method with other QA methods. Target structures used are $18 \mathrm{FM}$ targets in CASP7 and 12 FM targets in CASP8. See Tables 4 and 5 in Appendix for those targets. We should note that the 18 targets of CASP7 were already used to calibrate the FCS method in previous subsections, so that the targets of CASP7 are the training set and targets of CASP8 are the test set. Shown in Figures 2 are $\operatorname{GDTTS}(n, \mu)=\operatorname{GDTTS}\left(a^{\text {best }}(n, \mu), n\right)$, where the index $\mu$ distinguishes the FCS method and other QA methods developed by 22 QA teams in CASP7 (Figure 2a) and 41 QA teams in CASP8 (Figure 2b). The abscissa of Figure 2 represents the GDTTS of the best server model, i.e., the largest GDTTS of all server models for each target protein, GDTTS(n, best_server). From Figure 2, we find that in both CASP7 and CASP8, the FCS method is comparable with other well-performing QA methods though there are some examples for which the FCS method does not work well: Especially for the targets of GDTTS( $n$, best_server) > 55 in CASP7 (T0348 and T0350), the QA methods combined with the homology search for templates often give the better results than the FCS method which does not use a template. In this 
Table 1. Performance of LFCS in each model structure

For each of 4 example FM targets of CASP8, 5 models which have the highest FCS are selected. The correlation coefficient, $P_{\text {model }}$, is calculated by comparing LFCS and RMSD of every fragment in the model.

\begin{tabular}{c|ccc} 
Target & Model & $P_{\text {model }}$ & $\begin{array}{c}\text { GDTTS } \\
\text { of the model }\end{array}$ \\
\hline T0405_D1 & FALCON2 & 0.484 & 55.21 \\
& PSI4 & 0.484 & 55.21 \\
& PSI1 & 0.418 & 57.99 \\
& Zhang-Server2 & 0.383 & 41.67 \\
& Zhang-Server5 & 0.348 & 43.40 \\
\hline T0416_D2 & Zhang-Server5 & 0.603 & 66.23 \\
& SAM-T08-server2 & 0.473 & 36.84 \\
& fais-server5 & 0.353 & 52.19 \\
& Zhang-Server1 & 0.342 & 32.46 \\
& Zhang-Server3 & 0.257 & 34.65 \\
\hline T0443_D1 & MUFOLD-Server1 & 0.432 & 31.82 \\
& MUFOLD-Server4 & 0.431 & 31.82 \\
& Zhang-Server2 & 0.349 & 38.64 \\
& Zhang-Server4 & 0.295 & 53.41 \\
& Zhang-Server3 & 0.273 & 41.29 \\
\hline T0513_D2 & Zhang-Server2 & 0.710 & 51.45 \\
& FEIG3 & 0.621 & 44.20 \\
& Zhang-Server3 & 0.555 & 44.56 \\
& BAKER-ROBETTA4 & 0.537 & 43.84 \\
& MULTICOM-RANK5 & 0.264 & 40.94
\end{tabular}
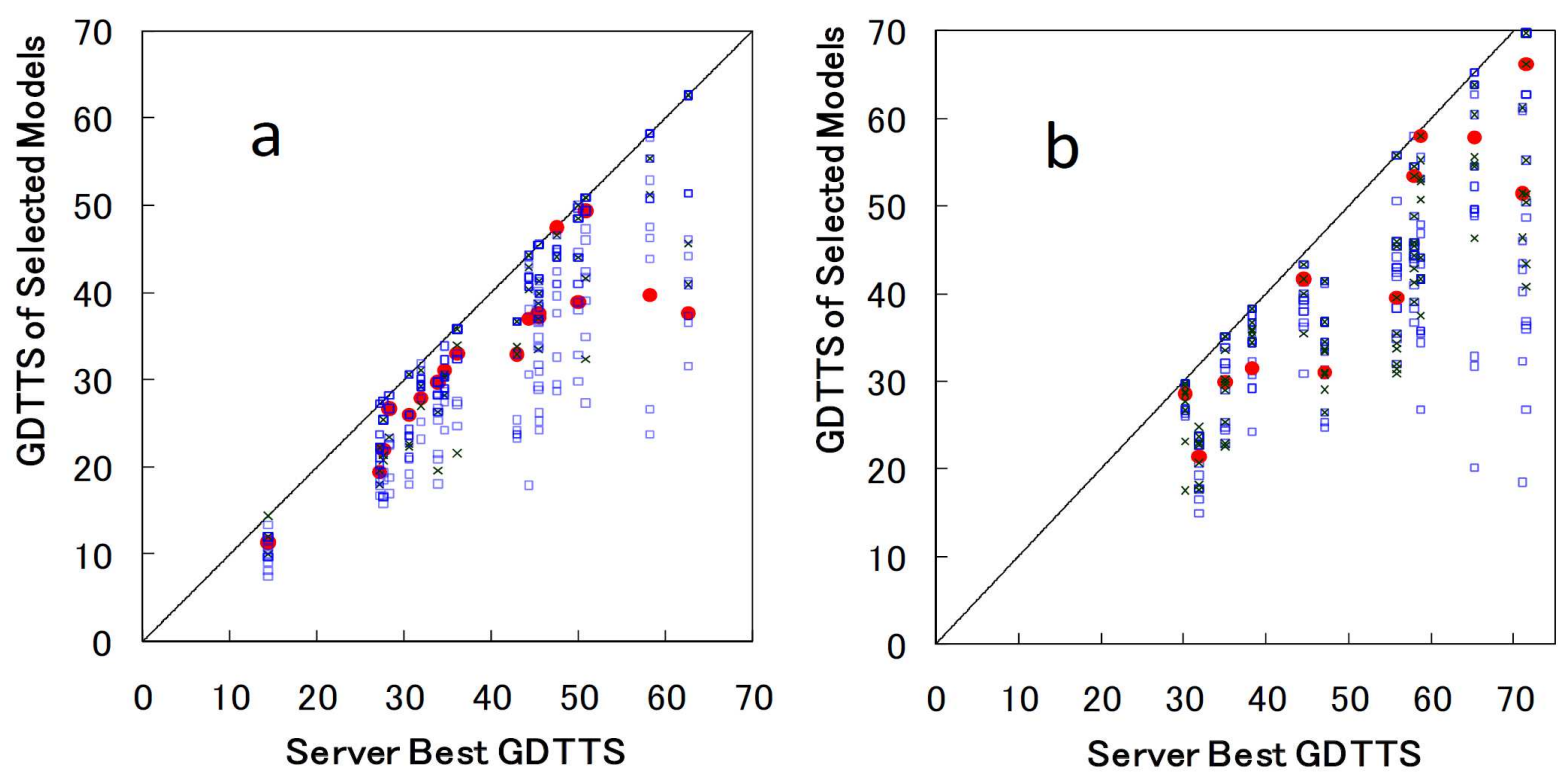

Figure 2. Comparison of the FCS method with other QA methods in terms of GDTTS

The FCS method and other QA methods are compared in terms of GDTTS of the best model selected by each QA method. The abscissa is the largest GDTTS of server models for each target. GDTTS obtained by the consensus based QA method (X), the FCS method (red filled circles), and the non-consensus-based QA methods $(\square)$ are shown for each target. Compared with the FCS method are the methods of QA proposed in (a) CASP7 and (b) CASP8. 

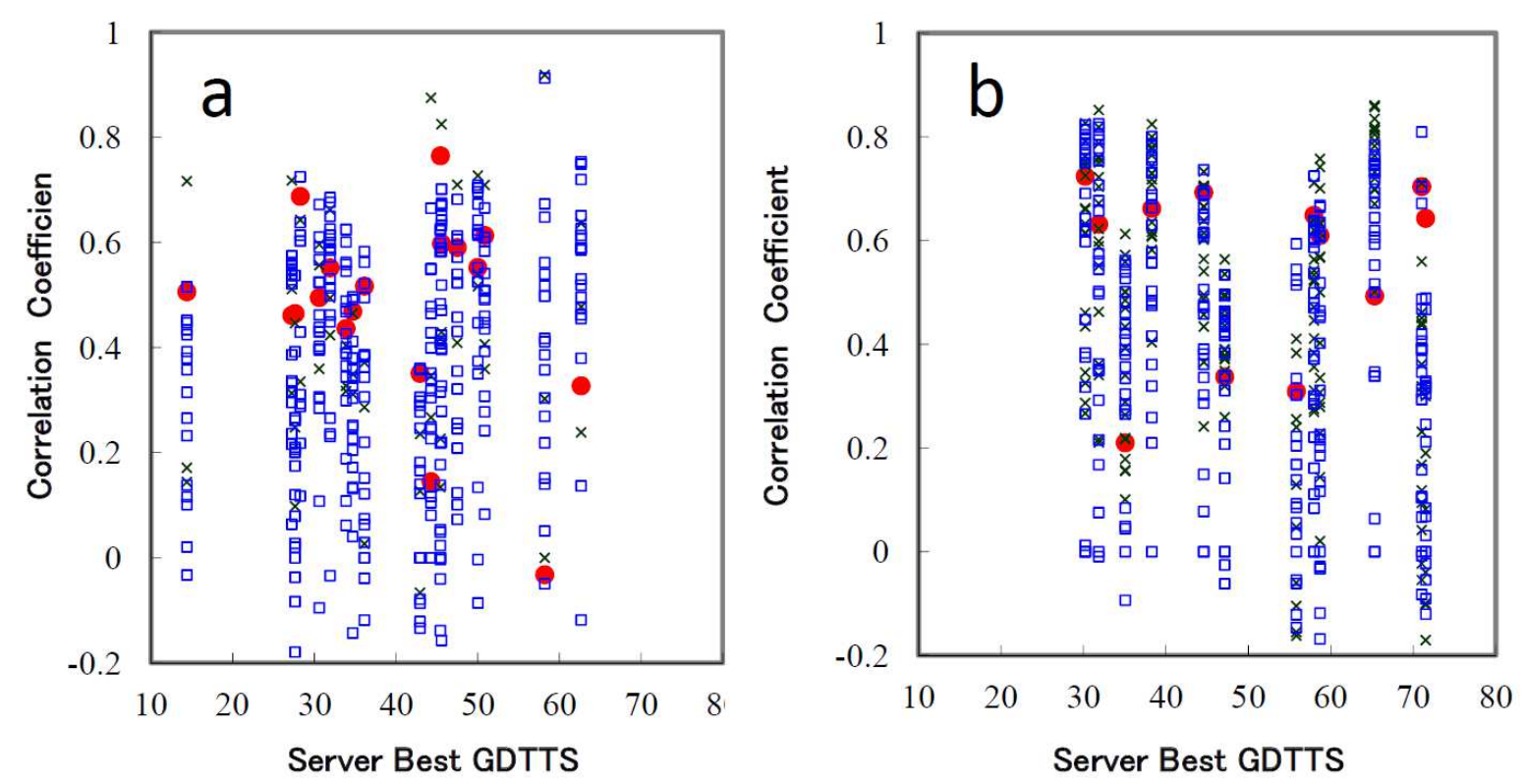

Figure 3. Comparison with other QA methods in terms of correlation coefficient

The FCS method and other QA methods proposed in CASP are compared in terms of the Pearson correlation coefficient between the QA score and GDTTS. The abscissa is the largest GDTTS of the models predicted by servers in (a) CASP7 and (b) CASP8. Correlation coefficients obtained by the consensus based QA method (x), the FCS method (red filled circle), and the non-consensus-based QA methods ( $\square)$ are shown for each target.

way, the FCS method compensates other methods, especially in application to the difficult targets.

The same results are obtained if we compare the ability of QA methods by another measure, $C C(n, \mu)$, as shown in Figure 3. Here, $C C(n, \mu)$ is the Pearson correlation coefficient between the score and GDTTS calculated for the ensemble of all available server models of each target: When we write the QA score calculated with the method $\mu$ for the model $a$ of the target $n$ as $\operatorname{Score}(a, n, \mu)$, $C C(n, \mu)$ is the correlation coefficient between $\operatorname{Score}(a, n, \mu)$ and $\operatorname{GDTTS}(a, n)$ for all $a$ at given $n$ and $\mu$. In CASP web pages, the correlation coefficient of the QA method is calculated by comparing the QA score of the whole protein structure with GDTTS of a partial domain of the model, so that we here made the same evaluation to obtain $C C(n, \mu)$ for the domain targets. Results of Figures 2 and 3 are summarized in Tables 2 and 3. From Table 2, we can see that $C C(n, F C S)$ is better than the average QA methods for many targets. As shown in Figure 3 and Table 2, the FCS method is comparable with other well-performing methods of QA especially for the targets of $\operatorname{GDTTS}(n$, best_server) < 55 in CASP7 (with exception of T0321_D2) and the targets in CASP8 (with a few exceptions, T0397_D1, T0476_D1, and T T0482_D1).

Table 3 is the comparison of FCS with several top QA methods used in CASP7 and CASP8. Particularly notable in Table 3 is the good performance of TASSER (ID\# 057 in CASP8) which uses the method similar to FCS [22]; In TASSER, the model fragment is assessed in terms of RMSD instead of the structural indices in our model, and together with such assessment of fragments, the consensus matching of the patterns of contacts among many models is also employed. We can see from Table 3 that TASSER gives consistently better results than FCS. A reason for this better performance might be due to the consensus technique used in TASSER, and 
Table 2. Summary of data shown in Figures 2 and 3

GDTTS of FCS and $C C(n, F C S)$ are compared with those averaged values over all QA methods and the best values of all QA methods for each target. Also shown is the rank of FCS among all QA methods used in each target. Numbers in bold are larger than the average QA results.

\begin{tabular}{|c|c|c|c|c|c|c|c|}
\hline \multicolumn{2}{|c|}{ Target } & \multirow{2}{*}{$\begin{array}{c}\text { GDTTS } \\
\text { FCS } \\
\text { (Rank/[\# of } \\
\text { QA teams]) } \\
\mathbf{2 6 . 7 1}(4 / 14)\end{array}$} & \multirow{2}{*}{$\begin{array}{c}\text { Averaged } \\
\text { GDTTS } \\
\text { of QA } \\
\text { methods }\end{array}$} & \multirow{2}{*}{$\begin{array}{c}\text { Best } \\
\text { GDTTS } \\
\text { of QA } \\
\text { methods } \\
28.26\end{array}$} & \multirow{2}{*}{$\begin{array}{c}\text { Pearson } \\
\text { FCS } \\
\text { (Rank/[\# of } \\
\text { QA teams]) } \\
\mathbf{0 . 6 8 8}(2 / 14)\end{array}$} & \multirow{2}{*}{$\begin{array}{c}\text { Averaged } \\
\text { Pearson } \\
\text { of QA } \\
\text { methods }\end{array}$} & \multirow{2}{*}{$\begin{array}{c}\text { Best } \\
\text { Pearson } \\
\text { of QA } \\
\text { methods }\end{array}$} \\
\hline CASP7 & T0287 & & & & & & \\
\hline & T0296 & $\mathbf{1 1 . 3 6}(12 / 22)$ & 10.93 & 14.37 & $0.506(3 / 22)$ & 0.272 & 0.717 \\
\hline & T0300 & $47.48(1 / 21)$ & 41.53 & 46.63 & $\mathbf{0 . 5 9 0}(5 / 21)$ & 0.375 & 0.710 \\
\hline & T0304 & $37.13(16 / 22)$ & 37.85 & 45.55 & $\mathbf{0 . 5 9 8}(10 / 22)$ & 0.507 & 0.825 \\
\hline & T0307 & $27.85(19 / 23)$ & 29.13 & 31.91 & $\mathbf{0 . 5 5 2}(12 / 23)$ & 0.497 & 0.685 \\
\hline & T0309 & $31.05(6 / 22)$ & 29.95 & 33.87 & $0.469(3 / 22)$ & 0.280 & 0.497 \\
\hline & T0314 & $25.97(5 / 23)$ & 24.40 & 30.58 & $\mathbf{0 . 4 9 5}(10 / 23)$ & 0.427 & 0.672 \\
\hline & T0316_D2 & $32.92(11 / 18)$ & 32.35 & 36.33 & $\mathbf{0 . 3 5 1}(6 / 24)$ & 0.110 & 0.360 \\
\hline & T0319 & $19.45(19 / 23)$ & 21.64 & 27.22 & $0.461(13 / 23)$ & 0.434 & 0.718 \\
\hline & T0321_D2 & $36.99(19 / 21)$ & 40.17 & 44.26 & $0.145(20 / 24)$ & 0.328 & 0.875 \\
\hline & T0347_D2 & $37.68(12 / 22)$ & 39.07 & 45.42 & $0.765(3 / 24)$ & 0.280 & 0.626 \\
\hline & T0348 & $39.75(20 / 22)$ & 51.23 & 58.19 & $-0.032(22 / 23)$ & 0.397 & 0.919 \\
\hline & T0350 & $37.64(20 / 23)$ & 52.05 & 62.64 & $0.327(20 / 23)$ & 0.508 & 0.753 \\
\hline & T0353 & $38.86(17 / 21)$ & 44.19 & 50.00 & $\mathbf{0 . 5 5 2}(11 / 22)$ & 0.504 & 0.727 \\
\hline & T0356_D1 & $21.98(12 / 22)$ & 22.48 & 27.62 & $0.465(3 / 24)$ & 0.111 & 0.537 \\
\hline & T0361 & $29.75(1 / 23)$ & 26.50 & 29.75 & $\mathbf{0 . 4 3 6}(10 / 23)$ & 0.407 & 0.625 \\
\hline & T0382 & $49.37(9 / 22)$ & 45.57 & 50.84 & $\mathbf{0 . 6 1 3}(3 / 22)$ & 0.459 & 0.709 \\
\hline & T0386_D2 & $33.02(14 / 22)$ & 33.08 & 35.80 & $\mathbf{0 . 5 1 7}(6 / 24)$ & 0.263 & 0.583 \\
\hline \multirow[t]{12}{*}{ CASP8 } & T0397_D1 & $29.88(17 / 35)$ & 30.13 & 35.06 & $0.210(34 / 42)$ & 0.350 & 0.613 \\
\hline & T0405_D1 & $57.99(1 / 41)$ & 44.46 & 57.99 & $\mathbf{0 . 6 1 0}(12 / 42)$ & 0.370 & 0.758 \\
\hline & T0405_D2 & $21.39(27 / 39)$ & 21.78 & 24.76 & $\mathbf{0 . 6 3 2}(20 / 42)$ & 0.552 & 0.852 \\
\hline & T0416_D2 & $66.23(13 / 36)$ & 57.12 & 69.74 & $\mathbf{0 . 6 4 3}(2 / 42)$ & -0.039 & 0.489 \\
\hline & T0443_D1 & $\mathbf{5 3 . 4 1}(8 / 40)$ & 46.52 & 57.95 & $0.649(5 / 42)$ & 0.460 & 0.725 \\
\hline & T0443_D2 & $41.67(8 / 40)$ & 40.72 & 43.33 & $\mathbf{0 . 6 9 3}(9 / 42)$ & 0.522 & 0.737 \\
\hline & T0465_D1 & $31.51(34 / 41)$ & 35.32 & 38.28 & $\mathbf{0 . 6 6 2}(23 / 42)$ & 0.626 & 0.824 \\
\hline & T0476_D1 & $31.03(31 / 39)$ & 34.94 & 41.38 & $0.337(34 / 42)$ & 0.384 & 0.564 \\
\hline & T0482_D1 & $\mathbf{5 7 . 8 4}(23 / 40)$ & 56.30 & 65.30 & $0.493(38 / 42)$ & 0.674 & 0.861 \\
\hline & T0496_D1 & $28.54(24 / 38)$ & 27.96 & 29.79 & $\mathbf{0 . 7 2 4}(20 / 42)$ & 0.597 & 0.826 \\
\hline & T0510_D3 & $39.53(20 / 40)$ & 40.99 & 55.81 & $\mathbf{0 . 3 0 9}(9 / 42)$ & 0.049 & 0.594 \\
\hline & T0513_D2 & $51.45(31 / 40)$ & 62.03 & 71.01 & $\mathbf{0 . 7 0 4}(4 / 42)$ & 0.315 & 0.810 \\
\hline
\end{tabular}


Table 3. Comparison of QA methods

For each QA method, the Pearson correlation coefficient and GDTTS averaged over assessed FM targets in CASP7 and CASP8 are compared.

\begin{tabular}{|c|c|c|c|c|c|c|c|c|c|}
\hline $\begin{array}{l}\text { QA team } \\
\text { in CASP7 }\end{array}$ & $\begin{array}{l}\text { ID \# of } \\
\text { the QA } \\
\text { team in } \\
\text { CASP7 }\end{array}$ & $\begin{array}{l}\text { Averaged } \\
\text { Pearson }\end{array}$ & $\begin{array}{c}\text { Averaged } \\
\text { GDTTS }\end{array}$ & $\begin{array}{c}\# \text { of } \\
\text { targets } \\
\text { assessed }\end{array}$ & $\begin{array}{l}\text { QA team in } \\
\text { CASP8 }\end{array}$ & $\begin{array}{l}\text { ID \# of } \\
\text { the QA } \\
\text { team in } \\
\text { CASP8 } \\
\end{array}$ & $\begin{array}{l}\text { Averaged } \\
\text { Pearson }\end{array}$ & $\begin{array}{c}\text { Averaged } \\
\text { GDTTS }\end{array}$ & $\begin{array}{c}\text { \# of } \\
\text { targets } \\
\text { assessed }\end{array}$ \\
\hline $\begin{array}{c}\text { Circle- } \\
\text { QA }\end{array}$ & 713 & 0.569 & 36.24 & 18 & Circle & 396 & 0.635 & 45.71 & 12 \\
\hline Pcons & 634 & 0.553 & 33.24 & 18 & ModFOLD & 199 & 0.607 & 44.22 & 12 \\
\hline ProQ & 633 & 0.508 & 35.26 & 18 & TASSER & 057 & 0.575 & 45.42 & 12 \\
\hline GeneSilico & 38 & 0.490 & 37.51 & 17 & $\begin{array}{c}\text { Pcons_ } \\
\text { ProQ }\end{array}$ & 469 & 0.567 & 41.79 & 12 \\
\hline $\begin{array}{l}\text { Ma- } \\
\text { OPUS }\end{array}$ & 91 & 0.484 & 34.91 & 18 & FCS & $\begin{array}{c}\text { present } \\
\text { work }\end{array}$ & 0.555 & 42.54 & 12 \\
\hline FCS & $\begin{array}{c}\text { present } \\
\text { work }\end{array}$ & 0.472 & 32.50 & 18 & Bilab-UT & 325 & 0.548 & 45.17 & 12 \\
\hline ABIpro-h & 699 & 0.469 & 37.72 & 18 & FAMSD & 140 & 0.523 & 42.55 & 12 \\
\hline $\begin{array}{c}\text { QA- } \\
\text { MODCHE } \\
\text { CK }\end{array}$ & 703 & 0.455 & 32.78 & 16 & $\begin{array}{l}\text { SAM-T08-M } \\
\text { QAU }\end{array}$ & 365 & 0.515 & 43.69 & 12 \\
\hline ProQlocal & 692 & 0.453 & 34.15 & 18 & $\begin{array}{l}\text { Fiser-QA- } \\
\text { COMB }\end{array}$ & 177 & 0.413 & 42.89 & 12 \\
\hline $\begin{array}{l}\text { CaspIta- } \\
\text { FRST }\end{array}$ & 717 & 0.390 & 32.61 & 17 & MULTICOM & 453 & 0.327 & 43.40 & 12 \\
\hline Bliab & 178 & 0.359 & 35.54 & 18 & DISTILLF & 117 & 0.307 & 40.80 & 12 \\
\hline $\begin{array}{c}\text { QA- } \\
\text { ModFOLD }\end{array}$ & 704 & 0.350 & 36.12 & 18 & $\begin{array}{c}\text { MODCHECK } \\
\text {-Jury }\end{array}$ & 052 & 0.266 & 36.38 & 12 \\
\hline LEE & 556 & 0.333 & 29.59 & 18 & LEE & 407 & 0.214 & 39.83 & 12 \\
\hline $\begin{array}{l}\text { Jones- } \\
\text { UCL }\end{array}$ & 13 & 0.310 & 32.47 & 16 & $\begin{array}{c}\text { MODCHECK } \\
-\mathrm{HD}\end{array}$ & 094 & 0.063 & 33.49 & 12 \\
\hline
\end{tabular}

the further comparison with the FCS method should provide a clue as to how to incorporate the consensus data into the present method.

\subsection{Modeling loops and coils}

It is interesting to examine whether the QA method proposed for the selection of the predicted models of the FM targets is also applicable to the TBM targets. Though shape and arrangement of secondary structures of templates are usually similar to those of the correct answer structures of TBM targets, the correct structures of irregular regions such as long loops connecting secondary structures or the coil regions near the termini are often quite different from those of the homologous templates. A most difficult part of the TBM problem is, therefore, to predict structures of these irregular regions [48][49][50]. Here, we apply the FCS method to selecting the model structures of such regions.

All targets of CASP8 were searched for the irregular regions longer than 10 residue length, which do not show a regular secondary structure in the correct answer conformations. 175 such regions were found, and structures of these 175 regions were difficult to predict: $70 \%$ of the server models had GDTTS < 70 for 70 regions among them. 
We calculate the fragment-based consistency score for the $\xi$ th region by

$$
F C S(\xi)=\sum_{i \in \operatorname{region}(\xi)} \operatorname{LFCS}_{i}
$$

By using this $F C S(\xi)$, we can select the model structures of GDTTS $>70$ for 13 regions, and we can further select 46 partial regions showing GDTTS >70. In Figures 4 and 5, we show several examples that the FCS method successfully select the best or the nearly best model structures of these regions.

Shown in Figures $4 \mathrm{a}-4 \mathrm{~d}$ are correlations between FCS and GDTTS of the example irregular regions. Correlation coefficient is large in Figures $4 \mathrm{a}$ and $4 \mathrm{c}$, and smaller in Figures $4 \mathrm{~b}$ and $4 \mathrm{~d}$. The FCS method, however, can select best models as shown in Figures 5a-5c and the 4th best model in the case of Figure 5d from all available server models. From Figure 5, we can see that these best models are still poor in quality without detailed agreement with the native structure, but the ability of the FCS method to select relatively good structures from the tested models implies that this method is a useful tool to improve prediction of loop and coil structures in the TBM problems.

\subsection{Ability to distinguish structures having small difference in sequence}

Among proteins categorized into the TBM targets in CASP8, especially to be mentioned are a pair of proteins, T0498 and T0499. T0498 and T0499 differ only in three residues from each other but have largely different conformations [42]. Since the sequence of T0498 is close to sequences of the already resolved proteins such as protein G, T0498 was categorized into the TBM target in
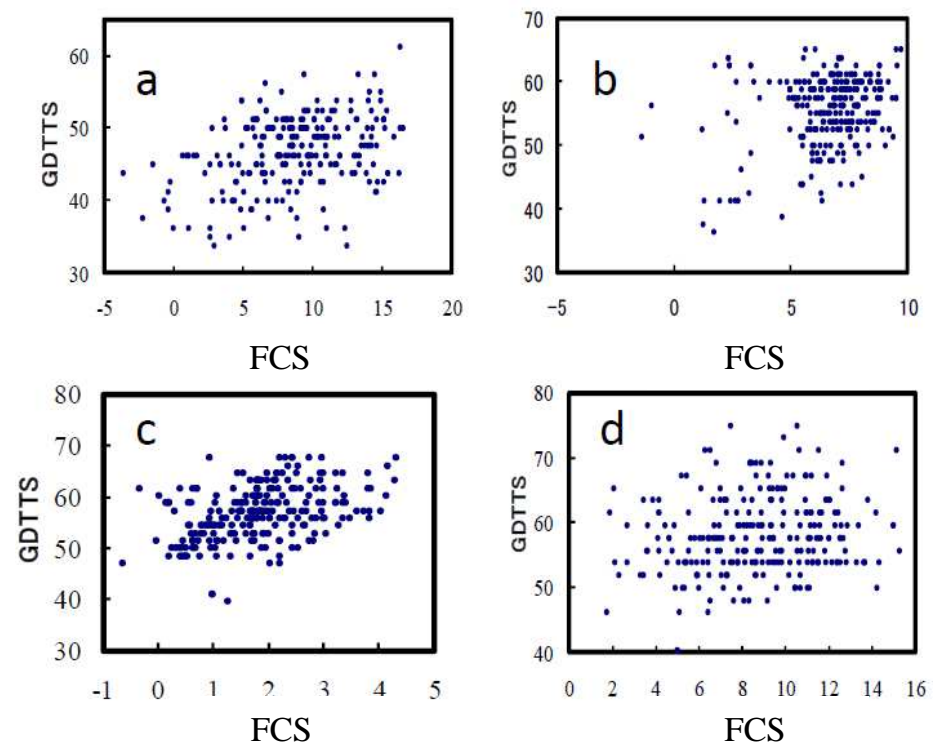

Figure 4. Quality assessment of model structures of irregular regions with the FCS method Quality of server models of loops and coils in the TBM targets of CASP8 are assessed by the FCS method. Correlation between FCS and GDTTS of model structures of those regions are shown for (a) 174-193th residue of the protein with the target number T0413 in CASP8 (PDB code: 3d0k), (b) 219-238th residue of T0427 (PDB code: 3d3y), (c) 1-17th residue of T0464 (PDB code: 2k5r), and (d) 73-85th residue of T0468 (PDB code: $2 \mathrm{k} 5 \mathrm{w}$ ). Correlation coefficients are 0.36 in $\mathbf{a}, 0.23$ in $\mathbf{b}$, 0.42 in $\mathbf{c}$, and 0.15 in $\mathbf{d}$. 


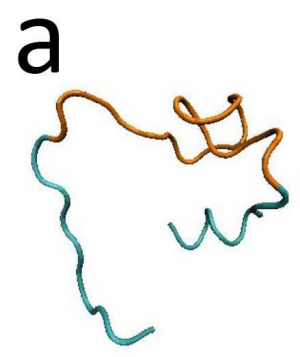

Native

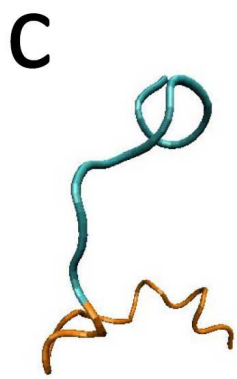

Native

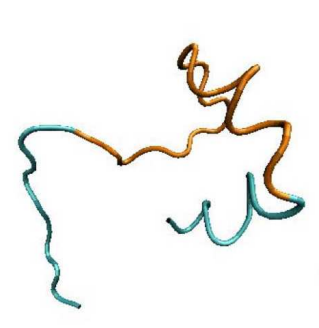

Selected

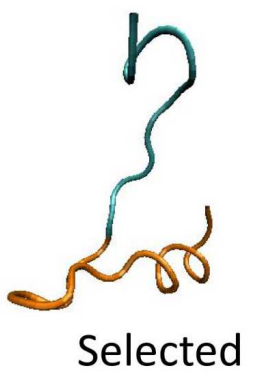

b
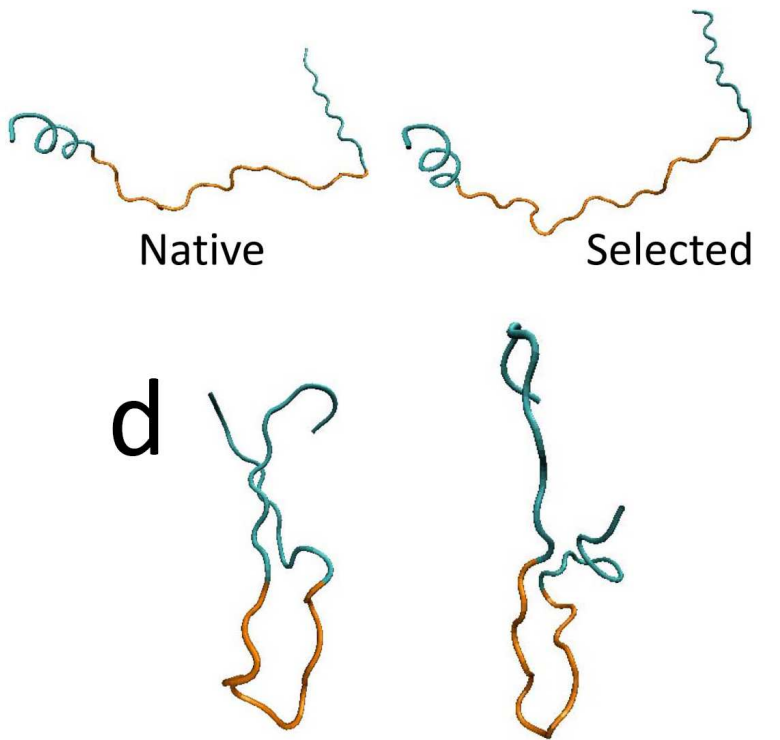

Native

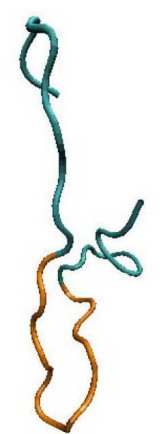

Selected

Figure 5. The model structures of irregular regions selected by the FCS method The best of server models of loops and coils selected by the FCS method are compared with the native structures. Quality of structures of the regions colored orange was assessed by the FCS method. Targets of a-d in this figure are same as those of a-d in Figure 4.

CASP, but its structural topology was different from those of assumed templates, so that the de novo prediction was practically necessary. Shown in Figures 6a and $6 \mathrm{~b}$ are FCS and GDTTS of all server models for T0498 and T0499, respectively. In both Figures 6a and 6b, results of server models are classified into two groups; the group of high GDTTS and the group of low GDTTS. We can find that the population of the low GDTTS group is much larger than the population of the high GDTTS group for T0498 in Figure 6a, so that the consensus-based QA completely fails to select a model structure from the high GDTTS group. The population of the high GDTTS group, on the other hand, is larger than that of the low GDTTS group for T0499 in Figure 6b, so that the consensus-based QA methods work for T0499. For T0498, structures of the high GDTTS group have helical structures and those of the low GDTTS group contain more sheet structure. For T0499, structures of the low GDTTS group are more helical than those of the high GDTTS group. A possible reason for such splitting of results into two groups is because each of these proteins can take two conformations of low free energy, one is helical and the other contains the sheet structure similar to protein $\mathrm{G}$ and the related proteins. Hence, it would be natural to assume that modification of the small number of residues changes the relative depth of these two free energy minima, leading to the change of balance of population of conformations, resulted in the different answers as target structures. Most of the server prediction methods in CASP8 relied on the sequence alignment among target and templates, so that the templates having similar structures to protein $G$ were selected for both T0498 and T0499 and the subtle change of the free energy depth could not be detected by those prediction methods. 

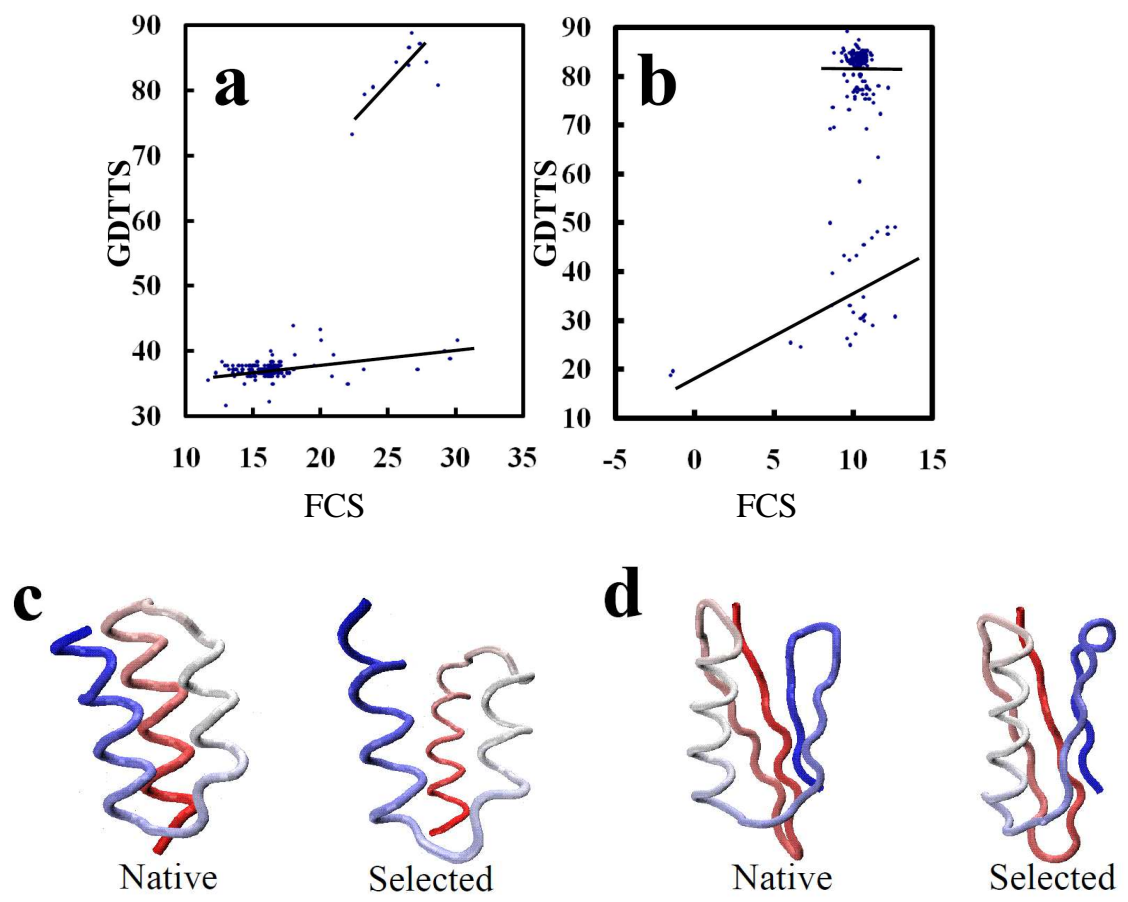

Figure 6. Distinction of different conformational topology of designed proteins having only three non-identical residues

GDTTS and FCS are shown in two-dimensional plots for all the model structures of T0498 and T0499 presented by servers in CASP8. GDTTS and FCS of 298 structures are shown for the target T0498 (a) and those of 268 structures are shown for the target T0499 (b). Gradient of lines in a and b represents the correlation coefficient between GDTTS and FCS. For T0498, the group of high GDTTS structures have the correlation coefficient 0.69 and the group of low GDTTS structures have the correlation coefficient 0.37 . For T0499, the group of high GDTTS structures have the correlation coefficient as small as 0.04 and the group of low GDTTS structures have the correlation coefficient 0.55. The experimentally observed native structure (left) and the best structure of 5 structures selected from all server models by the FCS method (right) are shown for T0498 (c) and T0499 (d). For T0498, the selected structure is 88.44 in GDT_TS and 1.49 $\AA$ in Root Mean Square Deviation (RMSD) from the observed structure. For T0499, the selected structure is 77.68 in GDTTS and $2.97 \AA$ in RMSD from the observed structure.

For T0498, FCS can distinguish the high GDTTS structures reasonably well from other sever models and shows the clear correlation between FCS and GDTTS for structures of the high GDTTS group. These features show that the FCS method works well to assess the model quality for T0498. For T0499, on the other hand, the FCS method does not select good models but only discriminates some wrong models. This is probably because the correct beta-sheet structure is not properly assessed with FCS, which suggests the direction of further improvement of the FCS method.

Shown in Figure 6c and 6d are structures selected with the FCS method. For T0498, the FCS method selected good models as shown in Fig.6c. For T0499, the FCS method selected both structures of the low GDTTS group and those of the high GDTTS group, but top five structures in FCS contain the structure in the high GDTTS group as shown in Fig.6d. Thus, the FCS method still has insufficient aspects, but further improvement of the FCS method should open the way toward the better QA from the direction which is not accessible with the consensus-based approaches. 


\section{Conclusion}

In this paper we developed a new QA method, the FCS method, to assess quality of the predicted model structures not only by the characteristics of individual residues but also by taking into account the local structural environment of fragments with the finite length. The present results showed that if the multi-residue features of the local structural environment of fragments are suitably averaged to derive the score of assessment, the FCS method is useful to improve the de novo prediction scheme. Indeed, the FCS method begins to be used to improve the performance of the dynamical fragment assembly method [51][52][53] for de novo prediction. The use of better fragments should further improve the FCS method, so that it would be interesting to see whether the physical refinement of fragments improves the score function. The FCS method also helps to select good models of long loops and to distinguish the artificially designed proteins which are only slightly different from each other in sequence but are largely different in conformation. These problems are difficult to treat with the consensus-based method, so that the suitably combined use of the present FCS method and the other consensus-based method should compensate each other for the further improvement of the model quality assessment techniques.

This work was partially supported by Theoretical and Computational Chemistry Initiative (TCCI) for the MEXT project of Strategic Programs for Innovative Research (SPIRE). 


\section{Appendix}

In this appendix we summarize the FM targets used in this paper; FM targets in CASP7 (Table 1) and FM targets in CASP8 (Table 2).

Table 4. FM target proteins in CASP7

\begin{tabular}{|c|c|c|c|c|c|}
\hline $\begin{array}{c}\text { Target } \\
\text { number in } \\
\text { CASP }\end{array}$ & $\begin{array}{l}\text { PDB } \\
\text { code }\end{array}$ & Type & $\begin{array}{l}\text { Size } \\
\text { (Number of } \\
\text { residues) }\end{array}$ & $\begin{array}{c}* * \text { The best } \\
\text { GDTTS of } \\
\text { server } \\
\text { models }\end{array}$ & $\begin{array}{c}* *, \# \text { Number } \\
\text { of server } \\
\text { teams }\end{array}$ \\
\hline T0287 & $2 \mathrm{~g} 3 \mathrm{v}$ & $\alpha$ & 161 & 28.26 & 166 \\
\hline T0296 & 2ha9 & $\alpha / \beta$ & 414 & 14.32 & 128 \\
\hline T0300 & $2 \mathrm{~h} 3 \mathrm{r}$ & $\alpha$ & 89 & 47.48 & 138 \\
\hline T0304 & $2 \mathrm{~h} 28$ & $\alpha+\beta$ & 101 & 45.55 & 184 \\
\hline T0307 & $2 \mathrm{~h} 5 \mathrm{n}$ & $\beta$ & 123 & 31.91 & 152 \\
\hline T0309 & $2 \mathrm{~h} 4 \mathrm{o}$ & $\alpha+\beta$ & 62 & 34.68 & 188 \\
\hline T0314 & 2hg6 & $\alpha+\beta$ & 103 & 30.58 & 161 \\
\hline *T0316_D2 & $2 \mathrm{hmA}$ & $\beta$ & 90 & 42.92 & 157 \\
\hline Т0319 & $2 \mathrm{j} 6 \mathrm{a}$ & $\alpha+\beta$ & 135 & 27.22 & 141 \\
\hline T0321_D2 & $2 \mathrm{~h} 1 \mathrm{q}$ & $\alpha / \beta$ & 156 & 44.26 & 145 \\
\hline T0347_D2 & $2 \mathrm{hwj}$ & $\alpha$ & 71 & 45.42 & 164 \\
\hline T0348 & $2 \mathrm{hf} 1$ & $\beta$ & 61 & 58.19 & 168 \\
\hline T0350 & $2 \mathrm{hc5}$ & $\alpha+\beta$ & 91 & 62.64 & 162 \\
\hline T0353 & $2 \mathrm{hfq}$ & $\alpha+\beta$ & 83 & 50.00 & 171 \\
\hline T0356_D1 & $2 \mathrm{idb}$ & $\alpha+\beta$ & 120 & 27.62 & 147 \\
\hline T0361 & $2 \mathrm{hkt}$ & $\alpha$ & 158 & 33.86 & 165 \\
\hline T0382 & $2 \mathrm{i} 9 \mathrm{c}$ & $\alpha$ & 119 & 50.84 & 175 \\
\hline T0386_D2 & $2 \mathrm{jk} 8$ & $\alpha+\beta$ & 81 & 36.11 & 139 \\
\hline
\end{tabular}


Table 5. FM target proteins in CASP8

\begin{tabular}{|c|c|c|c|c|c|}
\hline $\begin{array}{c}\text { Target } \\
\text { number in } \\
\text { CASP }\end{array}$ & $\begin{array}{l}\text { PDB } \\
\text { code }\end{array}$ & Type & $\begin{array}{c}\text { Size } \\
\text { (Number of } \\
\text { residues) }\end{array}$ & $\begin{array}{c}* * \text { The best } \\
\text { GDTTS of } \\
\text { server models }\end{array}$ & $\begin{array}{c}*{ }^{*}{ }^{\#} \text { Number } \\
\text { of server } \\
\text { teams }\end{array}$ \\
\hline *T0397_D1 & $3 d 4 r$ & $\beta$ & 82 & 35.06 & 200 \\
\hline T0405_D1 & - & $\alpha$ & 72 & 58.68 & 212 \\
\hline T0405_D2 & - & $\alpha+\beta$ & 208 & 31.85 & 195 \\
\hline T0416_D2 & $3 \mathrm{~d} 3 \mathrm{q}$ & $\alpha$ & 57 & 71.49 & 222 \\
\hline T0443_D1 & 3 dee & $\alpha$ & 66 & 57.95 & 216 \\
\hline T0443_D2 & 3 dee & $\alpha+\beta$ & 60 & 44.58 & 203 \\
\hline T0465_D1 & $3 \mathrm{dfd}$ & $\alpha$ & 96 & 38.28 & 223 \\
\hline T0476_D1 & $2 \mathrm{k} 5 \mathrm{c}$ & $\alpha+\beta$ & 87 & 47.13 & 208 \\
\hline T0482_D1 & $2 \mathrm{k} 4 \mathrm{v}$ & $\alpha+\beta$ & 67 & 65.30 & 214 \\
\hline T0496_D1 & 3do9 & $\alpha+\beta$ & 120 & 30.21 & 220 \\
\hline T0510_D3 & $3 \mathrm{doa}$ & $\alpha+\beta$ & 43 & 55.81 & 204 \\
\hline T0513_D2 & 3dup & $\alpha+\beta$ & 69 & 71.01 & 170 \\
\hline
\end{tabular}

\section{References}

[1] Krieger, E.; Joo, K.; Lee, J.; Lee, J.; Raman, S.; Thompson, J.; Tyka, M.; Baker, D.; Karplus, K. Proteins: Struct., Funct. Bioinform., 2009, 77, Suppl. 9, 114-122.

[2] Peng, J.; Xu, J. Proteins: Struct., Funct. Bioinform., 2011, 79, 1930-1939.

[3] Ben-David, M.; Noivirt-Birk, O.; Paz, A.; Prilusky, J.; Sussman, J. L.; Levy, Y. Proteins: Struct., Funct. Bioinform., 2009, 77, Suppl.9, 50-65.

[4] DeBartolo, J.; Colubri, A.; Jha, A. K.; Fitzgerald, J. E.; Freed, K. F.; Sosnick, T. R. Proc. Natl. Acad. Sci. USA, 2009, 106, 13748-13753.

[5] Voelz, V. A.; Shell, M. S.; Dill, K. A. PLoS Comput Biol., 2009, 5, e1000281.

[6] Papoian, G. A.; Ulander, J.; Eastwood, M. P.; Luthey-Schulten, Z.; Wolynes, P. G. Proc. Natl. Acad. Sci. USA, 2004, 101, 3352-3357. 
[7] Chikenji, G.; Fujitsuka, Y.; Takada, S. Proc. Natl. Acad. Sci. USA, 2006, 103, 3141-3146.

[8] Bartlett, G. J.; Taylor, W. R. Proteins: Struct., Funct. Bioinform., 2008, 71, 950-959.

[9] http://predictioncenter.gc.ucdavis.edu/

[10] Cozzetto, D.; Kryshtafovych, A.; Tramontano, A. Proteins: Struct., Funct. Bioinform., 2009, 77, Suppl.9, 157-166.

[11] Kihara, D.; Chen, H.; Yang, Y. D. Current Protein and Peptide Science, 2009, 10, 216-228.

[12] Kryshtafovych, A.; Fidelis, K. Drug Discovery Today, 2009, 14, 386-393.

[13] Ginalski, K.; Elofsson, A.; Fischer, D.; Rychlewski, L. Bioinformatics, 2003, 19, 1015-1018.

[14] Wallner, B.; Fang, H.; Elofsson, A. Proteins: Struct. Funct. Genet., 2003, 53, 534-541.

[15] Wallner, B.; Elofsson, A. Proteins: Struct. Funct. Bioinform., 2007, 69, 184-193.

[16] Larsson, P.; Skwark, M. J.; Wallner, B.; Elofsson, A. Proteins: Struct. Funct. Bioinform., 2009, 77, Suppl.9, 167-172.

[17] DeRonne, K. W.; Karypis, G. BMC Structural Biology, 2009, 9, 41.

[18] Archie, J. G.; Paluszewski, M.; Karplus, K. Proteins: Struct., Funct. Bioinform., 2009, 77, Suppl.9, 191-195.

[19] Benkert, P.; Künzli, M.; Schwede, T. Nucleic Acids Res., 2009, 37, W510-W514.

[20] Cheng, J.; Wang, Z.; Tegge, A. N.; Eickhol, J. Proteins: Struct. Funct. Bioinform., 2009, 77, Suppl.9, 181-184.

[21] McGuffin, L. J. Proteins: Struct. Funct. Bioinform., 2009, 77, Suppl.9, 185-190.

[22] Zhou, H.; Skolnick, J. Proteins: Struct. Funct. Bioinform., 2008, 71, 1211-1218.

[23] Wang, Z.; Tegge, A. N.; Cheng, J. Proteins: Struct. Funct. Bioinform., 2009, 75, 638-647.

[24] Eisenberg, D.; Lothy, R.; Bowie, J. Methods Enzymol., 1997, 277, 396-404.

[25] Zhou, H.; Zhou, Y. Proteins: Struct. Funct. Bioinform., 2004, 55, 1005-1013.

[26] Shirota, M; Ishida, T.; Kinoshita, K. Proteins: Struct. Funct. Bioinform., 2011, 79, 1550-1563.

[27] Sippl, M. J. Curr. Opin. Struct. Biol., 1995, 5, 229-235.

[28] Panchenko, A. R.; Marchler-Bauer, A.; Bryant, S. H. J. Mol. Biol., 2000, 296, 1319-1331.

[29] Wallner, B.; Elofsson, A. Protein Sci., 2003, 12, 1073-1086.

[30] Paluszewski, M.; Karplus, K. Proteins: Struct. Funct. Bioinform., 2009, 75, 540-549.

[31] Archie, J.; Karplus, K. Proteins: Struct. Funct. Bioinform., 2009, 75, 550-555.

[32] Kalman, M.; Ben-Tal, N. Bioinformatics, 2010, 26, 1299-1307.

[33] Rohl, C. A.; Strauss, C. E. M.; Misura, K. M. S.; Baker, D. Methods Enzymol., 2004, 383, 66-93.

[34] Bradley, P.; Misura, K. M. S.; Baker, D. Science, 2005, 309, 1868-1871.

[35] Lee, J.; Kim, S.-Y.; Lee, J. Biophys. Chem., 2005, 115, 209-214.

[36] Fujitsuka, Y.; Chikenji, G.; Takada, S. Proteins: Struct. Funct. Bioinform., 2006, 62, 381-398.

[37] Zhang, Y.; Arakaki, A. K.; Skolnick, J. Proteins: Struct. Funct. Bioinform., 2005, 61, 91-98.

[38] Zhou, H.; Skolnick, J. Biophys. J., 2007, 93, 1510-1518.

[39] Wu, S.; Skolnick, J.; Zhang, Y. BMC Biol., 2007, 5, 17.

[40] Terashi, G.; Takeda-Shitaka, M.; Kanou, K.; Iwadate, M.; Takaya, D.; Hosoi, A.; Ohta, K.; Umeyama, H. Proteins: Struct. Funct. Bioinform., 2007, 69, 98-107.

[41] Altschul, S. F.; Madden, T. L.; Schäffer, A. A.; Zhang, J.; Zhang, Z.; Miller, W.; Lipman, D. J. Nucleic Acids Research, 1997, 25, 3389-3402.

[42] Alexander, P. A.; He, Y.; Chen, Y.; Orban, J.; Bryan, P. N. Proc. Natl. Acad. Sci. USA., 2009, $106,21149-21154$.

[43] Available from: <http://www.ncbi.nlm.nih.gov/>

[44] Wang, G.; Dunbrack Jr. R. L., Bioinformatics, 2003, 19, 1589-1591.

[45] http://dunbrack.fccc.edu/PISCES.php

[46] http://www.predictioncenter.org/casp7/index.cgi 
[47] Zemla, A. Nucleic Acids Res., 2003, 31, 3370-3374.

[48] Arnautova, Y. A.; Abagyan, R. A.; Totrov, M. Proteins: Struct. Funct. Bioinform., 2011, 79, 477-498.

[49] Soto, C. S.; Fasnacht, M.; Zhu, J.; Forrest, L.; Honig, B. Proteins: Struct. Funct. Bioinform., 2008, 70, 834-843.

[50] Lee, J.; Lee, D.; Park, H.; Coutsias, E. A.; Seok, C. Proteins: Struct. Funct. Bioinform., 2010, 78, 3428-3436.

[51] Sasaki, T. N.; Cetin, H.; Sasai, M. Biochem. Biophys. Res. Comm., 2008, 369, 500-506.

[52] Sasaki, T. N.; Sasai, M. Chem. Phys. Lett., 2005, 402, 102-106.

[53] Lee, J.; Lee, J.; Sasaki, T. N.; Sasai, M.; Seok, C.; Lee, J. Proteins: Struct. Funct. Bioinform., 2011, 79, 2403-2417. 